\title{
Minireview \\ DNA photodamage recognition by RNA polymerase II
}

\author{
Florian Brueckner, Patrick Cramer* \\ Gene Center Munich, Department of Chemistry and Biochemistry, Ludwig-Maximilians-Universität München, \\ Feodor-Lynen-Strasse 25, D-81377 Munich, Germany \\ Center for integrated Protein Science CiPS ${ }^{M}$, Department of Chemistry and Biochemistry, Ludwig-Maximilians-Universität München, \\ Feodor-Lynen-Strasse 25, D-81377 Munich, Germany
}

Received 5 March 2007; accepted 6 May 2007

Available online 11 May 2007

Edited by Giulio Superti-Furga

\begin{abstract}
During gene transcription, RNA polymerase (Pol) II encounters obstacles, including lesions in the DNA template. Here, we review a recent structure-function analysis of Pol II transcribing DNA with a bulky photo-lesion in the template strand. The study provided the molecular basis for recognition of a damaged DNA by Pol II, which is the first step in transcription-coupled DNA repair (TCR). The results have general implications for damage recognition and the TCR mechanism.

(C) 2007 Federation of European Biochemical Societies. Published by Elsevier B.V. All rights reserved.
\end{abstract}

Keywords: Transcription-coupled DNA repair; Multisubunit cellular RNA polymerases; Structural biology of gene transcription

\section{Structural studies of Pol II elongation}

RNA polymerase (Pol) II synthesizes eukaryotic mRNA in the course of gene transcription. To ensure processive mRNA chain elongation, Pol II forms a stable elongation complex (EC) with transcribed DNA and product RNA. The X-ray structure of Pol II [1-5] enabled structural studies of Pol IInucleic acid complexes [6-11]. These studies elucidated many aspects of the molecular mechanism for mRNA chain elongation, and suggested how Pol II unwinds DNA, how it separates the RNA product from the DNA template, how it incorporates nucleoside triphosphate (NTP) substrates into the growing RNA chain, and how it can be inhibited by RNA (reviewed in part in $[12,13])$.

\section{Overcoming a natural obstacle during transcription}

Additional structural studies have elucidated how Pol II deals with a natural obstacle to transcription, intrinsic DNA arrest sites. At such sites, Pol II moves backwards along DNA and RNA, resulting in extrusion of the growing RNA $3^{\prime}$-end through the polymerase pore beneath the active site. Arrested polymerase cannot continue elongation by itself, but must be reactivated by mRNA cleavage. In such cases the very weak intrinsic RNA nuclease activity of Pol II is strongly stim-

\footnotetext{
"Corresponding author. Fax: +49 89218076999.

E-mail address: cramer@lmb.uni-muenchen.de (P. Cramer).
}

ulated by the extrinsic transcript cleavage factor TFIIS [14,15]. TFIIS inserts a hairpin into the polymerase pore and complements the active site with acidic residues, changes the enzyme conformation, and repositions the RNA transcript [7,16,17]. These studies suggested a "tunability" of the Pol II active site, which can apparently catalyze different reactions, including RNA synthesis and RNA cleavage. RNA cleavage creates a new $3^{\prime}$-end directly adjacent to the active site, from which transcription can resume, and the obstacle can be overcome.

\section{Damage recognition and TCR}

A recent study shows how Pol II deals with another obstacle, a bulky photo-lesion in the DNA template strand [18] (Fig. 1). The results not only elucidate an unexpected and complicated mechanism of damage recognition by Pol II, they also have implications for understanding how the damage is subsequently removed. Ultraviolet light damages DNA by inducing dimerization of adjacent pyrimidines in a DNA strand. The resulting cyclobutane pyrimidine dimer (CPD) lesions can block transcription and replication, and are a major cause of skin cancer [19]. Cells can eliminate CPDs slowly by genome-wide nucleotide excision repair (NER). For rapid and efficient repair, however, cells use a NER sub-pathway referred to as transcription-coupled DNA repair (TCR). TCR specifically removes lesions such as CPDs from the DNA strand transcribed by Pol II [20]. It is thought that only those lesions trigger TCR that can stably stall Pol II. CPDs are bulky lesions that lead to Pol II stalling, but other types of damages, such as oxidative damages, can be bypassed by Pol II, and would escape TCR [21]. Pol II stalling apparently triggers TCR by recruitment of a transcription-repair coupling factor $(\operatorname{Rad} 26 /$ CSB in yeast/human), and factors required for subsequent steps of nucleotide excision repair, including TFIIH, which unwinds DNA, and endonucleases, which incise the DNA strand on either side of the lesion [20,22-25]. The obtained DNA gap is subsequently filled by DNA synthesis and ligation [26,27].

\section{A tool box for studying damage recognition}

To study the mechanism of DNA damage recognition by Pol II, expertise in the synthesis of lesion-containing DNA (group of T. Carell at the University of Munich) was combined with expertise in preparing functional crystallization-grade ECs of 


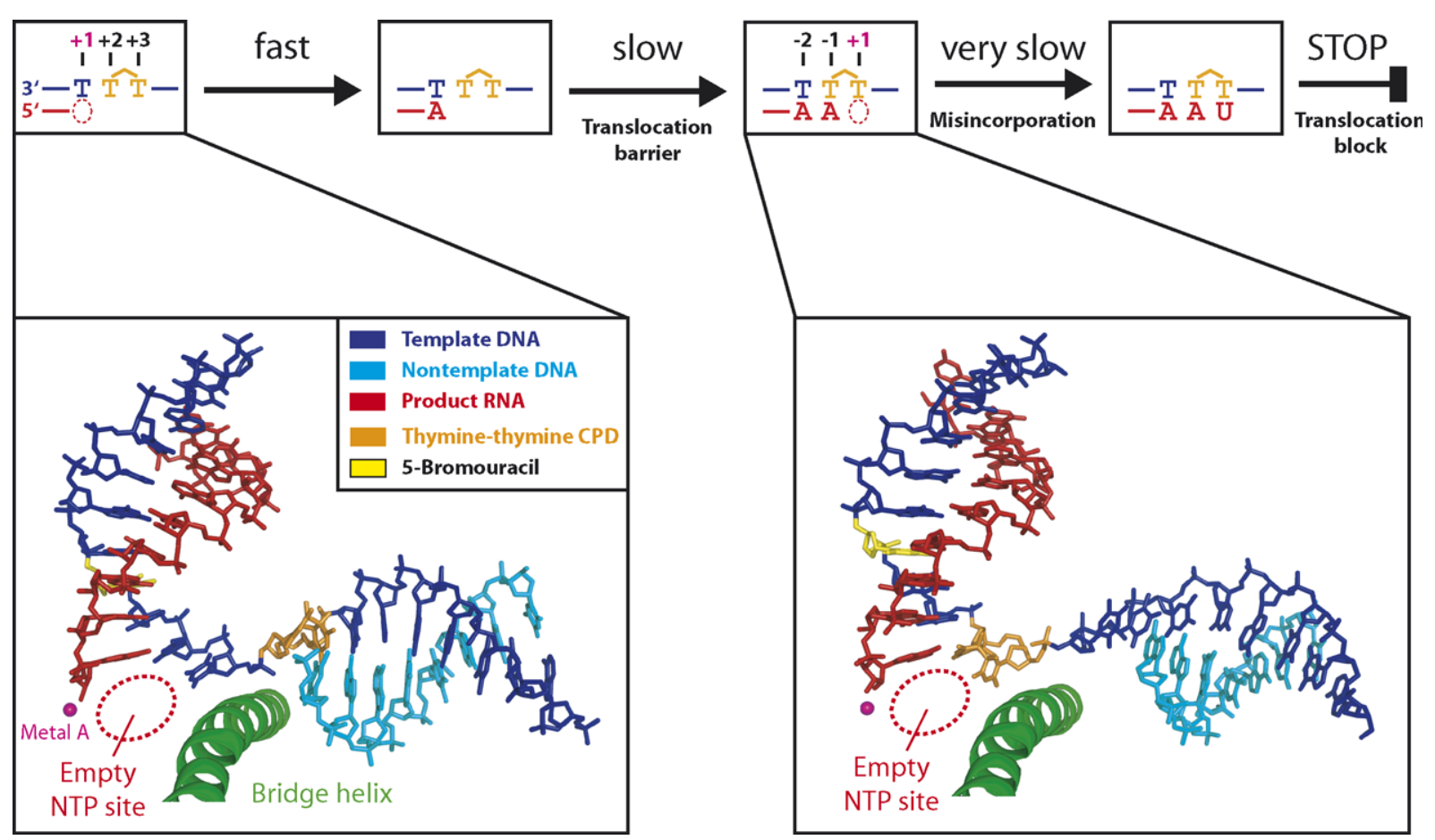

Fig. 1. Simplified mechanism of CPD DNA damage recognition by Pol II. On the top, a schematic is shown that depicts the last few steps before Pol II stalling. On the bottom, nucleic acids structures in Pol II ECs containing a thymine-thymine CPD lesion before (left) and in the active site (right) are shown. DNA template, DNA non-template, and RNA strands are in blue, cyan, and red, respectively. The CPD is shown as a stick model in orange. The active site magnesium ion (metal A) is depicted as a magenta sphere. Figures prepared with Pymol (www.pymol.org).

the complete 12-subunit Pol II (our group). CPD lesions were introduced into the DNA template strand at several different positions around the polymerase active site and the resulting Pol II ECs were studied structurally and in RNA elongation assays. The highly reproducible and clean system for reconstituting defined, fully functional Pol II ECs will be very useful for a detailed structure-function analysis of many aspects of the transcription mechanism in the future.

\section{The CPD lesion passes a barrier and enters the active site}

Pol II stalls when a CPD in the DNA template strand reaches the enzyme active site, after nucleotide incorporation opposite both CPD thymines [18,28,29]. However, it is not obvious how the CPD can reach the active site since transfer of a DNA template base from the downstream position +2 to the nucleotide insertion site at +1 over the polymerase bridge helix normally requires twisting of the base by $90^{\circ}$, and such twisting is not possible for the CPD thymines, since they are covalently linked. Indeed, the CPD is not stably accommodated at positions $+1 /+2$, and has to pass a translocation barrier to get into this position [18].

\section{The CPD induces misincorporation that stalls Pol II}

A CPD can be stably accommodated at positions $-1 /+1$ of the template strand in the active site of Pol II. Whereas Pol II correctly incorporates AMP opposite the $3^{\prime}$-thymine of the CPD, only UMP can be incorporated opposite the $5^{\prime}$-thymine $[18,29]$. The UMP misincorporation is very slow, and is the rate-limiting step in reaching the stalled state [18]. Specific UMP misincorporation may arise from the unusual location of the CPD $5^{\prime}$-thymine that adopts a wobble position with respect to the base in the undamaged complex [18]. The wobbled 5 -thymine could form two hydrogen bonds with UTP, but not with other NTPs. Pol II stalls because translocation of the CPD 5'-thymine-uracile mismatch base pair from position +1 to position -1 is strongly disfavored. This translocation event would move the damage-containing mismatch into the -1 position of the DNA-RNA hybrid, resulting in a distortion that likely destabilizes the EC [30]. Replacement of the misincorporated UMP by AMP in an artificial scaffold enables CPD bypass [18]. Thus, Pol II stalling requires CPD-directed misincorporation, and distortions due to the CPD alone are insufficient to cause Pol II stalling. Indeed, a T-U mismatch base pair alone was sufficient to stall the vast majority of Pol II complexes [18]. In contrast, DNA polymerases can correctly incorporate adenine opposite both CPD thymines, and, dependent on the type of polymerase, this can lead to stalling or lesion bypass $[31,32]$.

\section{A topological model for TCR}

What is the cellular signal that indicates to the repair machinery that a DNA lesion has been detected? In other words, how does the damage-stalled EC trigger assembly of the repair machinery? The lesion is deeply buried in the Pol II active site, thus it cannot be recognized by another factor. One possibility is that damaged DNA causes a conformational change in the Pol II EC that triggers recruitment of repair factors in an allosteric manner. However, the polymerase confor- 
1. CPD recognition

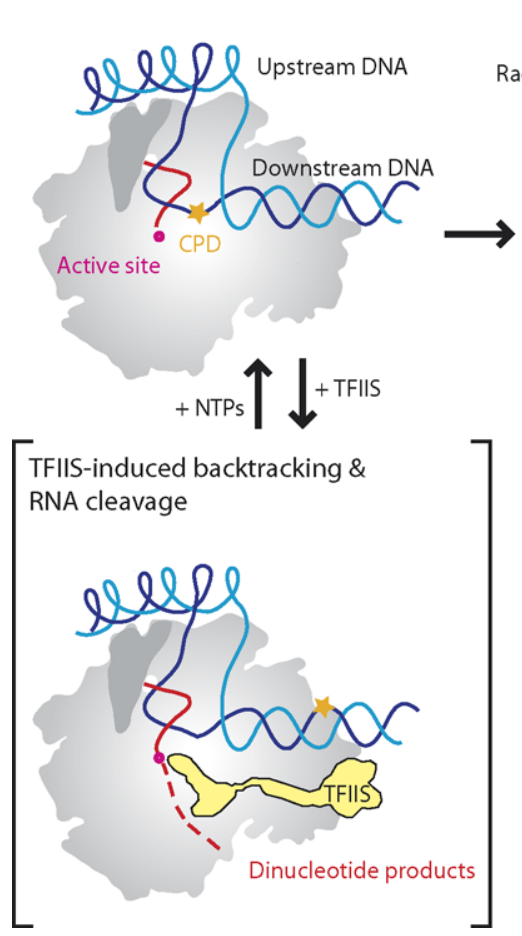

2. Complex maintenance \& bubble extension

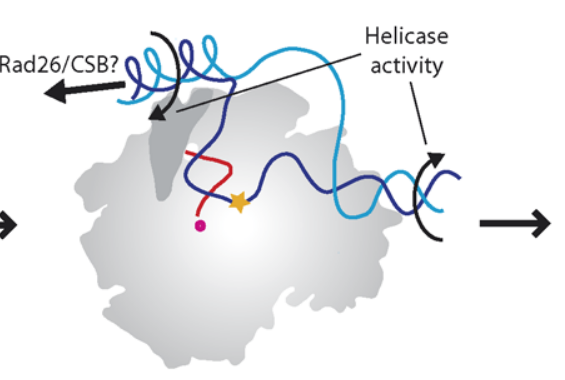

5. Gap repair \& Pol II recycling
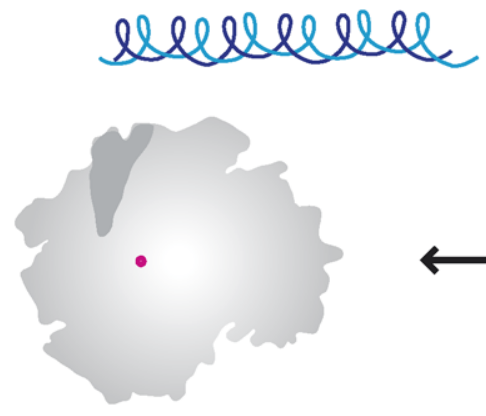

3.DNA dual incision

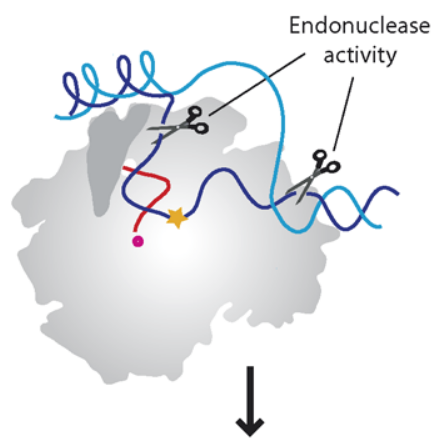

4. Removal of Pol II \& lesion

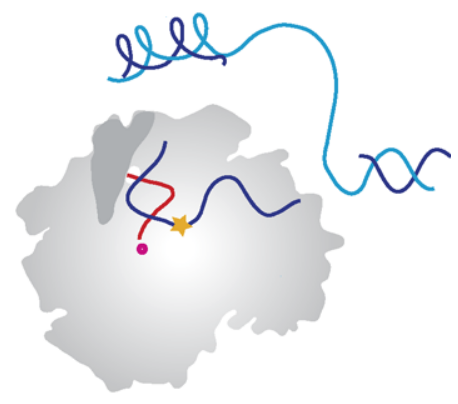

Fig. 2. Topological model for transcription-coupled DNA repair. The lesion-containing Pol II EC is shown schematically. The view and color code are as in Fig. 1. An orange star and a magenta sphere indicate the CPD lesion and the active site, respectively. The upstream DNA was placed on Pol II on the basis of its location in the bacterial RNA polymerase-promoter complex [38]. The non-template strand in the bubble region was modeled. TFIIH helicase subunits XPD and XPB may be involved in extending the transcription bubble in the upstream and downstream direction, respectively, to enable dual incision. Modeling shows that incisions could occur at the two edges of an extended bubble around 10-15 nucleotides upstream (3') and around 15-20 nucleotides downstream (5') of the lesion.

mation is unchanged in lesion-containing Pol II ECs [18]. This does not exclude that allostery exists and was simply not observed, but it argues against an allosteric model for TCR. In addition, all observations are consistent with excision of a lesion-containing DNA fragment in the presence of Pol II. Such a model for eukaryotic TCR combines and extends previous models $[24,33,34]$ and can explain recognition of the stalled complex without allostery or exposure of the lesion (Fig. 2). At a CPD lesion, CSB can counteract TFIIS-induced backtracking [35,36], resulting in a stably stalled complex. This may open a time window for assembly of the repair machinery. TFIIH may then catalyze extension of the transcription bubble, permitting dual incision of the template strand on the Pol II surface [23,24]. The lesion-containing DNA fragment and the RNA transcript could then be removed together with Pol II, although dual incision is apparently not sufficient to achieve this [24]. The remaining gapped DNA is repaired. Pol II may be recycled, circumventing its ubiquitination and destruction [37].

\section{Conclusions and perspectives}

The new study shows how a cellular RNA polymerase recognizes a DNA photo-lesion, and explains why the polymerase stalls when it encounters the lesion. The new data also suggest a preferred model for TCR that can be tested experimentally. In addition, the study predicts that DNA damages that have the potential to direct misincorporation can generally stall
Pol II and trigger TCR. However, given the unexpectedly complicated mechanism of CPD recognition by Pol II, recognition of different types of lesions must be studied in a detailed manner both structurally and functionally before general conclusions can be drawn. Finally, there are many open questions on TCR events subsequent to damage recognition, and it will require a highly interdisciplinary approach to address these experimentally.

\section{References}

[1] Cramer, P. et al. (2000) Architecture of RNA polymerase II and implications for the transcription mechanism. Science 288 (5466), 640-649.

[2] Cramer, P., Bushnell, D.A. and Kornberg, R.D. (2001) Structural basis of transcription: RNA polymerase II at 2.8 angstrom resolution. Science 292 (5523), 1863-1876.

[3] Armache, K.J., Kettenberger, H. and Cramer, P. (2003) Architecture of the initiation-competent 12-subunit RNA polymerase II. Proc. Natl. Acad. Sci. USA 100, 6964-6968.

[4] Bushnell, D.A. and Kornberg, R.D. (2003) Complete RNA polymerase II at 4.1 A resolution: implications for the initiation of transcription. Proc. Natl. Acad. Sci. USA 100, 6969-6972.

[5] Armache, K.J. et al. (2005) Structures of complete RNA polymerase II and its subcomplex Rpb4/7. J. Biol. Chem. 280, 71317134 .

[6] Gnatt, A.L. et al. (2001) Structural basis of transcription: an RNA polymerase II elongation complex at $3.3 \AA$ resolution. Science 292 (5523), 1876-1882.

[7] Kettenberger, H., Armache, K.J. and Cramer, P. (2004) Complete RNA polymerase II elongation complex structure and its interactions with NTP and TFIIS. Mol. Cell 16, 955-965. 
[8] Kettenberger, H. et al. (2006) Structure of an RNA polymerase II-RNA inhibitor complex elucidates transcription regulation by noncoding RNAs. Nat. Struct. Mol. Biol. 13 (1), 44- 48.

[9] Westover, K.D., Bushnell, D.A. and Kornberg, R.D. (2004) Structural basis of transcription: separation of RNA from DNA by RNA polymerase II. Science 303 (5660), 1014-1016.

[10] Westover, K.D., Bushnell, D.A. and Kornberg, R.D. (2004) Structural basis of transcription: nucleotide selection by rotation in the RNA polymerase II active center. Cell 119 (4), 481-489.

[11] Wang, D. et al. (2006) Structural basis of transcription: role of the trigger loop in substrate specificity and catalysis. Cell 127 (5), 941-954.

[12] Armache, K.J., Kettenberger, H. and Cramer, P. (2005) The dynamic machinery of mRNA elongation. Curr. Opin. Struct. Biol. 15, 197-203

[13] Boeger, H. et al. (2005) Structural basis of eukaryotic gene transcription. FEBS Lett. 579 (4), 899-903.

[14] Fish, R.N. and Kane, C.M. (2002) Promoting elongation with transcript cleavage stimulatory factors. Biochim. Biophys. Acta 1577 (2), 287-307

[15] Wind, M. and Reines, D. (2000) Transcription elongation factor SII. Bioessays 22 (4), 327-336.

[16] Kettenberger, H., Armache, K.J. and Cramer, P. (2003) Architecture of the RNA polymerase II-TFIIS complex and implications for mRNA cleavage. Cell 114, 347-357.

[17] Cramer, P. (2004) RNA polymerase II structure: from core to functional complexes. Curr. Opin. Genet. Dev. 14 (2), 218-226.

[18] Brueckner, F. et al. (2007) CPD damage recognition by transcribing RNA polymerase II. Science 315 (5813), 859-862.

[19] Mitchell, J.R., Hoeijmakers, J.H. and Niedernhofer, L.J. (2003) Divide and conquer: nucleotide excision repair battles cancer and ageing. Curr. Opin. Cell. Biol. 15 (2), 232-240.

[20] Saxowsky, T.T. and Doetsch, P.W. (2006) RNA polymerase encounters with DNA damage: transcription-coupled repair or transcriptional mutagenesis? Chem. Rev. 106 (2), 474-488.

[21] Charlet-Berguerand, N. et al. (2006) RNA polymerase II bypass of oxidative DNA damage is regulated by transcription elongation factors. EMBO J. 25 (23), 5481-5491.

[22] Svejstrup, J.Q. (2002) Mechanisms of transcription-coupled DNA repair. Nat. Rev. Mol. Cell Biol. 3 (1), 21-29.

[23] Selby, C.P. et al. (1997) RNA polymerase II stalled at a thymine dimer: footprint and effect on excision repair. Nucleic Acids Res. 25 (4), 787-793.
[24] Tremeau-Bravard, A. et al. (2004) Fate of RNA polymerase II stalled at a cisplatin lesion. J. Biol. Chem. 279 (9), 7751-7759.

[25] Mu, D. and Sancar, A. (1997) Model for XPC-independent transcription-coupled repair of pyrimidine dimers in humans. J. Biol. Chem. 272 (12), 7570-7573.

[26] Sancar, A. (1996) DNA excision repair. Annu. Rev. Biochem. 65, 43-81.

[27] Prakash, S. and Prakash, L. (2000) Nucleotide excision repair in yeast. Mutat. Res. 451 (1-2), 13-24.

[28] Tornaletti, S. et al. (1997) Nucleotide sequence context effect of a cyclobutane pyrimidine dimer upon RNA polymerase II transcription. J. Biol. Chem. 272, 31719-31724.

[29] Mei Kwei, J.S. et al. (2004) Blockage of RNA polymerase II at a cyclobutane pyrimidine dimer and 6-4 photoproduct. Biochem. Biophys. Res. Commun. 320 (4), 1133-1138.

[30] Kireeva, M.L. et al. (2000) The 8-nucleotide-long RNA:DNA hybrid is a primary stability determinant of the RNA polymerase II elongation complex. J. Biol. Chem. 275 (9), 6530-6536.

[31] Li, Y. et al. (2004) Nucleotide insertion opposite a cis-syn thymine dimer by a replicative DNA polymerase from bacteriophage T7. Nat. Struct. Mol. Biol. 11 (8), 784-790.

[32] Ling, H. et al. (2003) Replication of a cis-syn thymine dimer at atomic resolution. Nature 424 (6952), 1083-1087.

[33] Sarker, A.H. et al. (2005) Recognition of RNA polymerase II and transcription bubbles by XPG, CSB, and TFIIH: insights for transcription-coupled repair and Cockayne Syndrome. Mol. Cell 20 (2), 187-198.

[34] Svejstrup, J.Q. (2003) Rescue of arrested RNA polymerase II complexes. J. Cell. Sci. 116 (Pt 3), 447-451.

[35] Laine, J.P. and Egly, J.M. (2006) Initiation of DNA repair mediated by a stalled RNA polymerase IIO. EMBO J. 25 (2), 387397.

[36] Selby, C.P. and Sancar, A. (1997) Cockayne syndrome group B protein enhances elongation by RNA polymerase II. Proc. Natl. Acad. Sci. USA 94 (21), 11205-11209.

[37] Woudstra, E.C. et al. (2002) A Rad26-Def1 complex coordinates repair and RNA pol II proteolysis in response to DNA damage. Nature 415 (6874), 929-933.

[38] Murakami, K.S. et al. (2002) Structural basis of transcription initiation: an RNA polymerase holoenzyme-DNA complex. Science 296 (5571), 1285-1290. 